\title{
Numerical Investigations of Transient Flow Characteristic in Axial Flow Pump and Pressure Fluctuation Analysis Based on the CFD Technique
}

\author{
Ahmed Ramadhan Al-Obaidi* and Amer A. Mohammed \\ Mechanical Engineering Department / College of Engineering / Mustansiriyah University Baghdad / Iraq,
}

Received 29 September 2018; Accepted 28 November 2019

\begin{abstract}
The technique of Computational fluid dynamics was applied to investigate flow field analysis including static pressure, axial, radial and tangintial velocities vectors as well as fluctuations of pressure analyses in axial pump. Pressure fluctuations are the core causes increase noise level and vibration. For studying pressure mechanism fluctuation in pump changed monitoring rotating points are stalled at an impeller blade. Standard k- epsilon turbulence model and sliding mesh technique are adopted by using CFD. The numerical investagation found that pressure in an axial flow pump rises near inlet impeller region to outer part. Higher value of pressure was take place at impeller outlet region. Also, the computational analysis noted that the when a pump functions in larger mass flow, the area of high-pressure in impeller outlet decreases. Moreover, under low flow rate $(5 \mathrm{l} / \mathrm{min})$ in middle surface the high-speed area appears near or closed blade and blade tip region for all velocities under investigations. Furthermore. fluctuations of pressure for different point under different flows has four valleys and peaks the similar number of axial impeller passages blades. The results foun that there are two imprtant regions the first one ispositive and second one is negative pressures, this happends owing to this negative pressure area has lower pressure value. Therefore, due to the decline in the water pressure in this area may cause cavitation.
\end{abstract}

Keywords: Axial flow pump, Static pressure and velocity variations, Pressure fluctuation, CFD

\section{Introduction}

Axial pump is used in many industry and domestic applications, these types of pumps are suffered from different kinds of problems such as vibration and noise causes by complex pump geometry shape, flow conditions and cavitation phenomenon. Due to these problems, the pump performance and life of the pumps will decrease. The failure of pump will be becoming more and increase at high flow rate, high reliability, large capability and high speed. However, important reason that causes the pump structural failure is unsteady flow. Therefore, in order to enhance the pump stability and increase the life of a pump at changed conditions the study on flow filed within an axial flow pump [1-2] is very important to help for preventing of fatigue damage and enhancement the pump stability. Many studies have been studied on the flow behaviour analysis as well the effect of geometrical configurations for axial flow pumps [3$5]$ in recent years. There are different researches attempt to analyse on flow field in axial pump. Shi et al. [6] studied flow behaviour of axial pump numerically and then validated with experimental test. Meng et al. [7] numerically analysed the fluid structure in unstable area for the reversible axial impeller pump.

Pan et al. [8] researched the pressure fluctuation in the axial pump by using mean decomposition approaches. Song et al. [9] analysed the impact of vortex on pressure fluctuation in the system of axial pump using CFD analysis. Bosioc et al. [10] analysed hydrodynamic flow within axial large storage

*E-mail address: ahmedram@uomustansiriyah.edu.iq ISSN: 1791-2377 @ 2019 School of Science, IHU. All rights reserved. doi:10.25103/iestr.126.09 pump. They manufactured the experimental rig using stainless steel and used with two different tanks, diameters of outlet and inlet tubes were 0.08 and $0.1 \mathrm{~m}$. Gauge transducer pressure at the inlet was able to measure between -1 to +2.5 bars and the outlet was range between 0 to 6 bar. The results have shown that the minimum pressure was increased and moved from leading region impeller edge blades to an inducer. Also, when used the axial rotor the deviation angle is enhanced about $40 \%$. Moreover, more uniform flow field was appeared at impeller of a pump when a rotor was installed upstream of the pump blades to the inducer domain. Cheng et al. [11] Researched the transient region for the mixed flow and axial flow. Vibration and noises generated within the different of pumps when they operated at unstable operating region. They have found that the unsteady state flow in pump dividing to two parts: firstly, due to external conditions and secondly by self-flow excitation. Also, latter types can combine and caused flow induced due to vibration and vortex train caused due to the change of the shape geometric. Moreover, the experimental data shown that the main inner hydraulic losses can cased the unstable flow conditions in the pump. In addition, the rotating stall flow in an impeller and recirculation flow at the pump inlet occur due to guide vane mixed flow pump. Pan et al. [12] compared hydraulic performance for mixed flow and axial flow. The design parameters they used the impeller diameter 300mm, pump rotation speed of $1450 \mathrm{rpm}$, the designed flow condition was $0.347 \mathrm{~m}^{3} / \mathrm{s}$, head design of $9.5 \mathrm{~m}$, and specific speed of 576 . The results found that the length of long axial and configuration of compact radial regions were for axial flow area at a part vane. Mixed flow in the part volute was a vertical reliability and profile of robust pancake. Also, under designing operating condition axial rotor was with good 
performance of a pump. Whereas, impellers of mixed flows have a greater zone of maximum efficiency. Furthermore, an impeller was a better suction performance at operating design condition. Tao et al. [13] Investigated the sweep blade hydrodynamics within a hi-speed impeller axial fuel pump. They studied three hydrodynamics performance types first one is straight, second one is positive, and third one is negative of sweep axial blade in fuel pump through modelled three kinds of impellers. The outside diameter of $70 \mathrm{~mm}$, impeller involves from 4 blades, the pump rotating speed of $8000 \mathrm{rpm}$ and rate of mass flow was $10.75 \mathrm{~kg} / \mathrm{s}$. As a result, they have found that vortex flow happens near the inlet blade surface and it increases closed to the tip blade area. Also, it can be seen that the blade type positive sweep was effective for decreasing hydraulic flow losses due to driving flow stalled fluid in the middle part of impeller blade. In addition, when using positive sweep blade type the instability operating zone can be reduced. Furthermore, using latter type of blade can offer an effective means for security and stability in different types of axial fuel pump. Li et al. [14] investigated complicated fluid of flow numerically within a rotor pump. They simulated model for rate of flow was $305 \mathrm{~kg} / \mathrm{s}$, head of $30 \mathrm{~m}$, speed was about $3000 \mathrm{rpm}$ and SST k- $\omega$ model was used for simulation calculations. Because of complexities of setting up the turbulent flow conditions just velocity direction and rate of mass flow were imposed. Therefore, it can be predictable that a flow was fully flow developed through the more interactions flow between the wall and flow. Results have shown that the greater flow velocity was at the inlet part of impeller. The great appearance of a recirculation and incidence zone was happened at inlet part closed the impeller leading district edge. Also, the pressure at impeller inlet region was augmented near impeller leading area edge to the trailing region edge, due to flow acceleration caused more turbulent vortex flow structures on an impeller as well as near out guide region vane.

In current analysis, CFD method is employed to numerically investigate flow trend inside a pump and to predict the pump performance at variety of conditions. The 3D computational flow domains, numerical setup and boundary conditions have been described. Computational data were validated with the experimental pump results. However, the mechanisms cause change in static pressure, velocity variations, axial, radial, tangential velocities, and pressure fluctuations under different conditions have not yet been clarified. Therefore, the outcomes of CFD investigation in this study reveals the above parameters that change the performance of axial pump. Using CFD method can offer more details regarding qualitative and quantitative data inside a pump for instance flow behaviour, pressure, flow velocity and pressure in time domain investigations. So, this work provides good information for engineers to enhance a axial pump performance.

\section{Numerical Simulation Technique}

In turbo machinery industries the CFD analysis techniques are being extensively employed, for investigating and designing of objects for example compressors, turbines, and variety types of pumps. Moreover, with CFD methods to investigate flow pattern inside pump allows the engineering designers to obtain more qualitative and quantitative information regarding conditions behaviour systems. Evidently, CFD method can be utilized to enhance and decline the pump design cost. Outcomes of numerical calculations can offer sensibly accurate data about the trend of fluid flow in types of pumps. The flow pattern inside pumps, mostly in blade channels have a significant impact on the pump performance. Though, achieving experiments to investigate the flow trend can prove to be complex. This brief approach comprises four important steps, first one contains of pump geometry preparation parts; pump geometry has three flow domains axial impeller, inlet and outlet tubes. The second methodology step includes generating meshing domain for each portion geometry. Third one is important step in this method is setting of solver setup. Such step providing that flow properties types, chosen variety of boundary conditions, type of turbulence model selecting, mesh parts interfaces, numerical flow schemes and convergence setting controls. The final step is very important one to choose different types of parameters for monitoring flow then run computational calculations for FLUENT and collect stored results the throughout post processor period. Entire the above steps are proven in Figure 1.

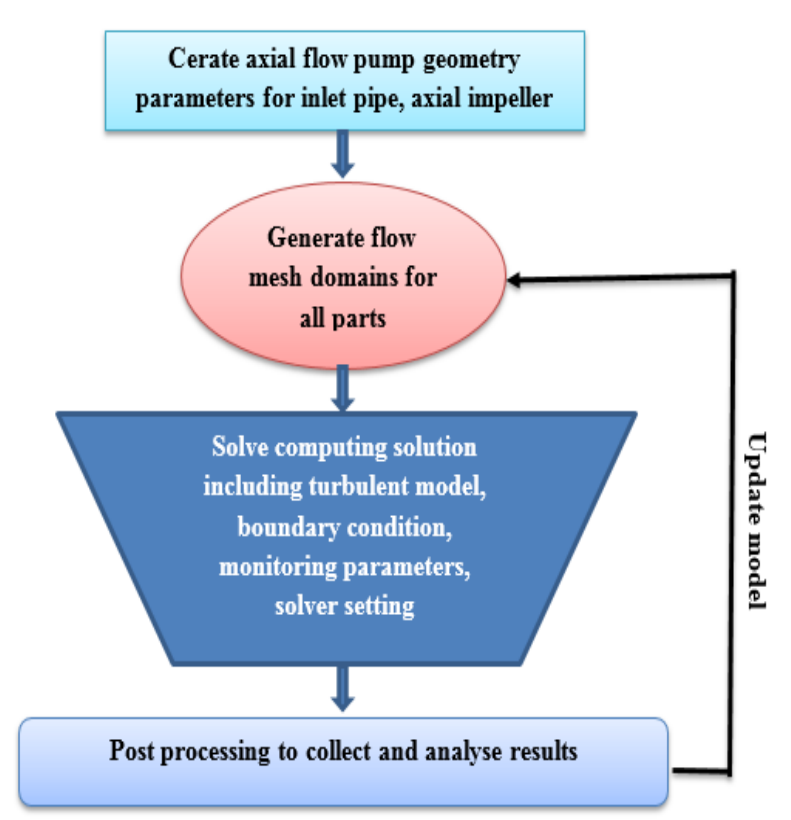

Fig. 1. Steps of computational methodology using CFD technique

In this work, in order to valid the axial pump numerical data, closed flow loop rig done by Mostafa, N. H. [15] have been selected as the research object. The experimental test for the axial flow pump was offered with a different set of equipment and instruments with uncertainty value for each instrument as described at Table 1. Furthermore, specifications of a pump are listed in Table 2 [15]. The speed conditions of the pump was by control panel.

Table 1. Different types of devices and uncertainty values

\begin{tabular}{c|c|c|c}
\hline Apparatus & Variable & Unit & $\begin{array}{c}\text { Uncertainty } \\
\text { value }\end{array}$ \\
\hline Water meter & $\begin{array}{c}\text { Rate of } \\
\text { flow } \\
\text { Voltage }\end{array}$ & $\begin{array}{c}\text { Volt }(\mathrm{V}) \\
\text { bar })\end{array}$ & $\begin{array}{c} \pm 0.709 \\
\pm 0.050\end{array}$ \\
$\begin{array}{c}\text { Pressure } \\
\text { transducer } \\
\text { Ammeter }\end{array}$ & $\begin{array}{c}\text { Pressure } \\
\text { Current }\end{array}$ & $\begin{array}{c}\text { Ampere } \\
(\mathrm{A})\end{array}$ & \pm 0.50 \\
$\begin{array}{c}\text { Flow } \\
\text { tachometer }\end{array}$ & $\begin{array}{c}\text { Impeller } \\
\text { speed } \\
\text { Meter of torque }\end{array}$ & $\begin{array}{c}\text { Revelation } \\
\text { per min } \\
(\mathrm{N})\end{array}$ & \pm 30.11 \\
\hline
\end{tabular}


Table 2. Values and specifications of the pump

\begin{tabular}{c|c|c}
\hline Parameter & Value & Unit \\
\hline Design speed & 3000 & $(\mathrm{rpm})$ \\
Hub diameter & 50 & $(\mathrm{~mm})$ \\
Tip diameter & 102 & $(\mathrm{~mm})$ \\
Number of blades & 4 & $(-)$ \\
Hub to tip ratio & 0.495 & $(-)$ \\
Motor power & 2.2 & $(\mathrm{~kW})$ \\
Blade angles & 60 & $($ degree $)$ \\
\hline
\end{tabular}

2.1. Computational Flow domains for the Axial Pump and Boundary Conditions

In this study, four-blade axial flow pump have been employed to generate the three dimensions impeller as depicts in Figure 2. The numerical simulations applying CFD methods are carried out with a sliding mesh technique for muddling the rotor part. impeller of axial flow pump is solved in a rotating part and the inlet and outlet pipes are fixed parts. The impeller diameter of $101 \mathrm{~mm}$, the blade angle was 60 . The boundary condition simulation is set at the inlet part with variety of inlet velocity, and the outflow is set as outlet condition [16-20]. The pump experimental data are got from Mostafa, N. H. [15] as mentioned earlier. The turbulence model has been modelled with epsilon k- $\varepsilon$ model.

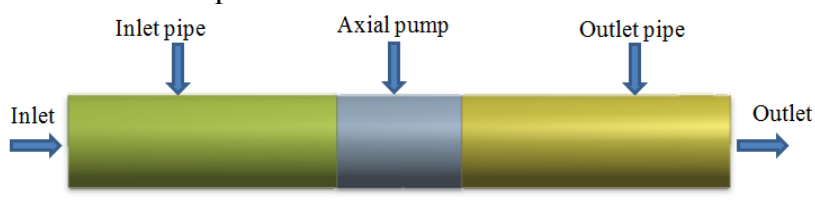

(a)

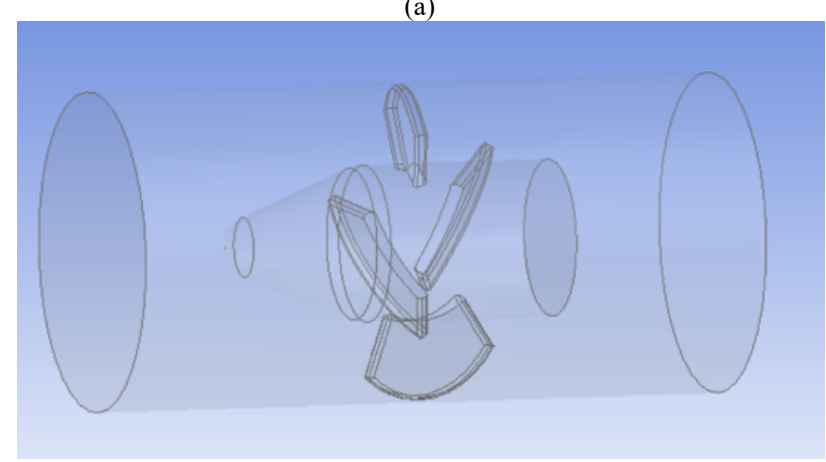

(b)
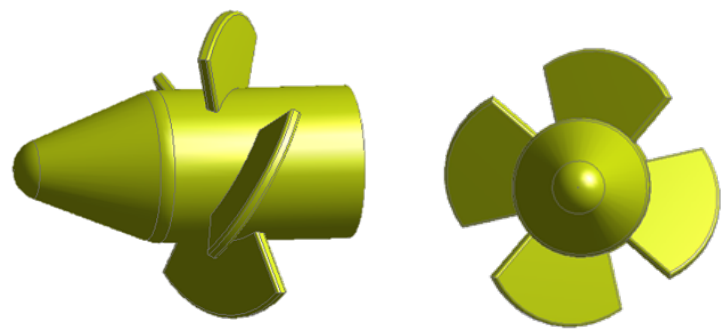

(c)

Fig. 2. Three dimensions model of impeller and axial flow pump domains, (a) Computational flow domains for the axial pump, (b) Pump domain and(c) Impeller domain

\subsection{Meshing Generation of Flow Domains}

The flow mesh generation domains requests to be spatially discretised for different number of very small sections, these is named the mesh flow element, the equations of liquid flow used to solve on them. In this computational investigation is applied the unstructured tetrahedral type flow meshing domain owing to irregular profile of geometric and more excessive mixing in an axial impeller. Additionally, type of dense mesh is used to capture the effects of near-wall on fluid flow. For an impeller and other regions mesh with size of $1.6 \mathrm{~mm}$ is specified. Moreover, to find the optimal flow mesh tests of different mesh independences are carried out to capture accurate prediction of flow domain, the elements of mesh are presented in Figure 3.
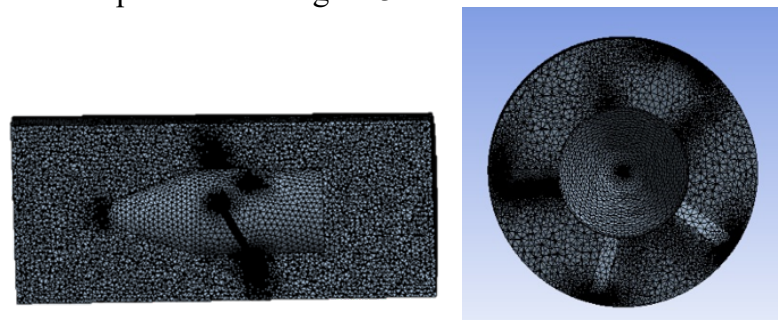

Fig. 3. Axial flow pump mesh

\section{Computational Flow Field Analysis}

To investigate the flow behaviour in a pump, CFD numerical transient analysis is used to predict the pump performance at altered conditions. To study the impact of flow interaction between pipe wall and axial impeller in numerical calculations a sliding mesh technique take into account. Details information about pump results are shown in the next section.

\subsection{Mesh Independence Test}

Three flow meshes are tested in this work in this study three meshes involving one, two, and three million elements are chosen, as exhibited in Table 3 . The results for changing the difference pressure are lower than 3\% for different meshes. Mesh with 2 million elements is chosen for further investigation in a pump due to it is capable to obtain accurately predict results.

Table 3. Test of independence mesh results in the pump

\begin{tabular}{c|c|c}
\hline $\begin{array}{c}\text { Mesh elements } \\
\text { no. }\end{array}$ & $\begin{array}{c}\text { Difference } \\
\text { pressure }\end{array}$ & $\begin{array}{c}\text { Difference in } \\
\text { pressure }\end{array}$ \\
\hline & $($ bar) & $(\%)$ \\
$1 \mathrm{M}$ & 0.272 & \\
$2 \mathrm{M}$ & 0.293 & 7.17 \\
$3 \mathrm{M}$ & 0.301 & 2.66 \\
\hline
\end{tabular}

\subsection{Test of Time Steps Independence (TSI)}

The TSI leads to increase accurate outcomes by using CFD methods [21-22]. Table shows the 3 different time steps. For further analysis $2.77778 \times 10^{-4}(\mathrm{~s})$ time step was selected.

Table 4. TSI results of the pump

\begin{tabular}{c|c|c}
\hline T S & $\begin{array}{c}\text { Difference } \\
\text { pressure }\end{array}$ & $\begin{array}{c}\text { Difference in } \\
\text { pressure }\end{array}$ \\
\hline$(\mathrm{s})$ & $($ bar) & $(\%)$ \\
$5.55556 \times 10^{-5}$ & 0.268 & \\
$1.66667 \times 10^{-4}$ & 0.285 & 5.96 \\
$2.77778 \times 10^{-4}$ & 0.293 & 2.73 \\
\hline
\end{tabular}

\subsection{Results Validation}

Validation results are the most important stage in numerical analysis to verify accurately computational physical model in actual flow system [16-20]. The experimental work carried out by Mostafa, N. H. was used to validate the numerical model in the current study [15]. Figure 4 represents the difference pressure for both transient numerical results using 
CFD and experiments condition data for variety of flows. As noted that there is a a well arrangement between two results.

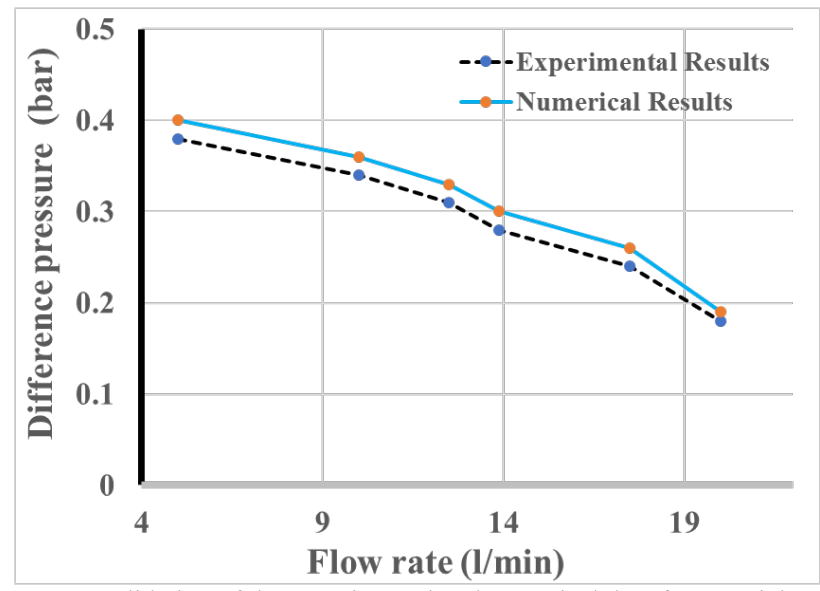

Fig. 4. Validation of the experimental and numerical data for an axial flow pump

In this study, different typical axial flow pump operation conditions of low flow condition $(5 \mathrm{l} / \mathrm{min})$, design condition of $12.5 \mathrm{l} / \mathrm{min}$ and high rate of flow condition $20 \mathrm{l} / \mathrm{min}$ are used to investigated the flow field analysis in a pump as represented in next sections.

\subsection{Distributions of Pressure and Velocity in a Pump}

In order to obtain good understanding regarding characteristics, investigation of flows in the pump pressure and velocity variations are discussed. To analyse the change of velocity and pressure in the axial flow pump domain and meridional interface was chosen.

Figure 5 illustrates the variations in pressure of the pump with impeller rotor blades is four. Diameter of impeller used of $102 \mathrm{~mm}$, pump speed $3000 \mathrm{rpm}$ with different mass flows including a) 5 , b) design rate 12.5 and c) high flow conditions $20 \mathrm{l} / \mathrm{min}$. The entire contours of static pressures in this figure are plotted on a same scale for precise comparison analyses. As perceived that the static pressure distributions at various flows have the similar tendency. Pressure changes and increases from impeller inlet to outer region. In addition, the higher value of pressure take place near the outer impeller diameter. Also, results observed that pressure at impeller pressure blade side was more than impeller blade side suction. Moreover, at high rate of flow pressure in the pump parts decrease. Therefore, depend on latter above results it can be found that the rate of flow was highly effect on flow pattern in the pump especially on static pressure variations.

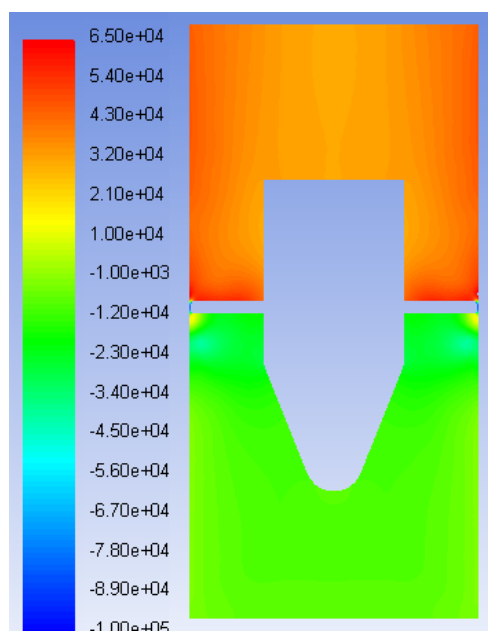

(a)

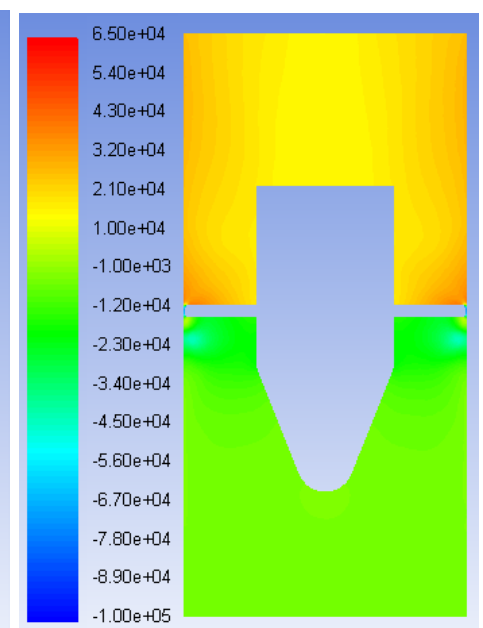

(b)

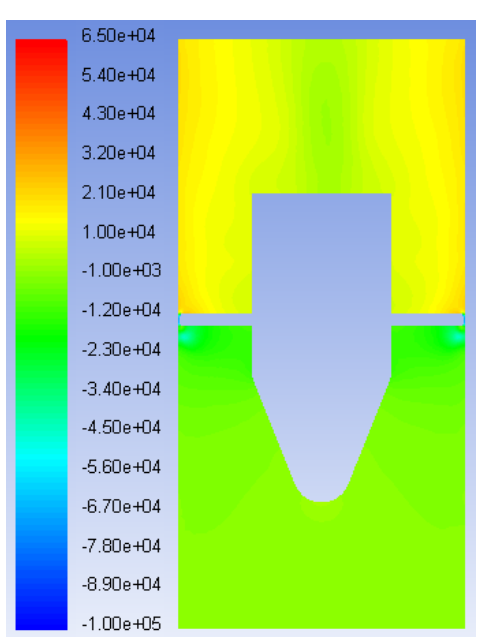

(c)

Fig. 5. Middle surface of static pressure variations at changed flows

Figure 6 shows the velocity variations at centre surface of (A) axial, (B) radial and (C) tangential velocities vectors) in a pump with 4 blades of impeller. Impeller diameter is $102 \mathrm{~mm}$ and speed is $3000 \mathrm{rpm}$ at the same above figure conditions. The contours of velocity (A) axial, (B) radial and (C) tangential shown in this figure are the similar condition scale. With low flow ( $5 \mathrm{l} / \mathrm{min}$ ) in middle surface the high-speed area appears near or closed blade and blade tip region for all velocities under investigations (A) axial, (B) radial and (C) tangential these areas were affected by guide vines. The minimum and maximum of velocities vectors were alternating shown at the middle section. Also, maximum velocities were near impeller edge blades. Additionally, it is perceived that secondary flows along the radial direction between blades, and it leads to cause low efficiency of the pump. Furthermore, the numerical results have shown that the flows are becoming uneven with low flow rate, which means that the axial flow pump functions well at high flow rate conditions. Whereas, the pump work not so well at low rate of flow.

To more search in flow behaviour for providing further details regarding pressure variations alteration. The pressure variations on the pump are calculated at nine different surface locations separately and are named S1 to S9 at nine different cross sections at the front and rear of an impeller as represents in Figure 7. 


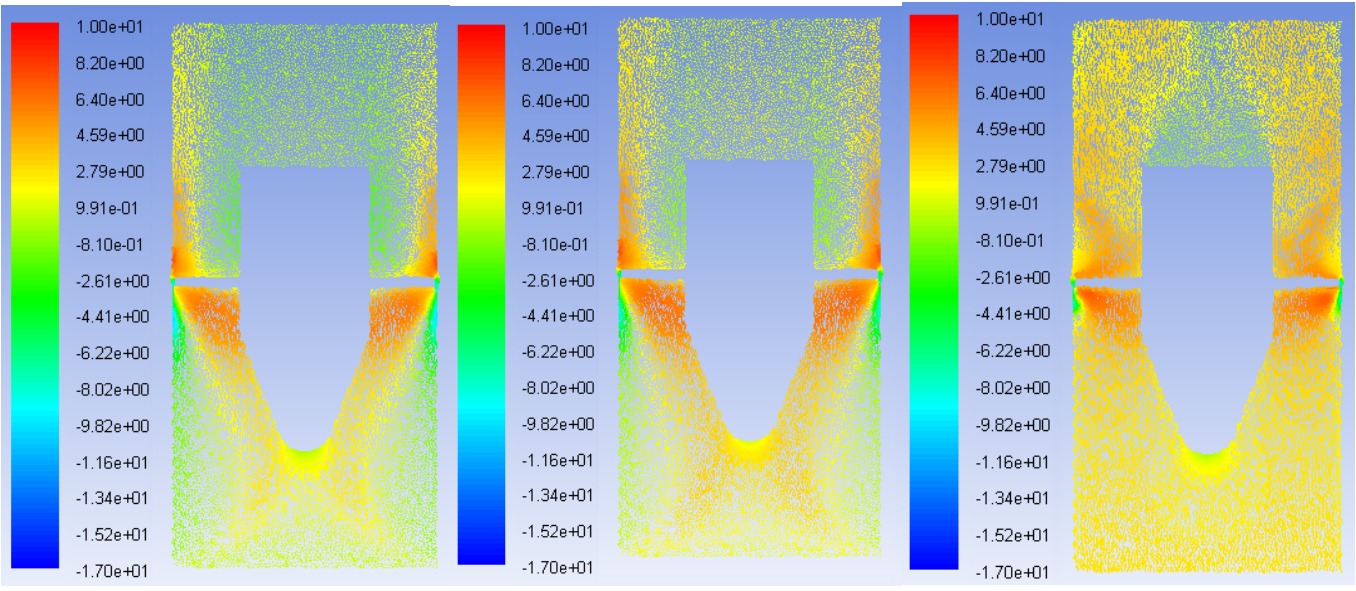

(A) Middle surface of axial velocity vectors

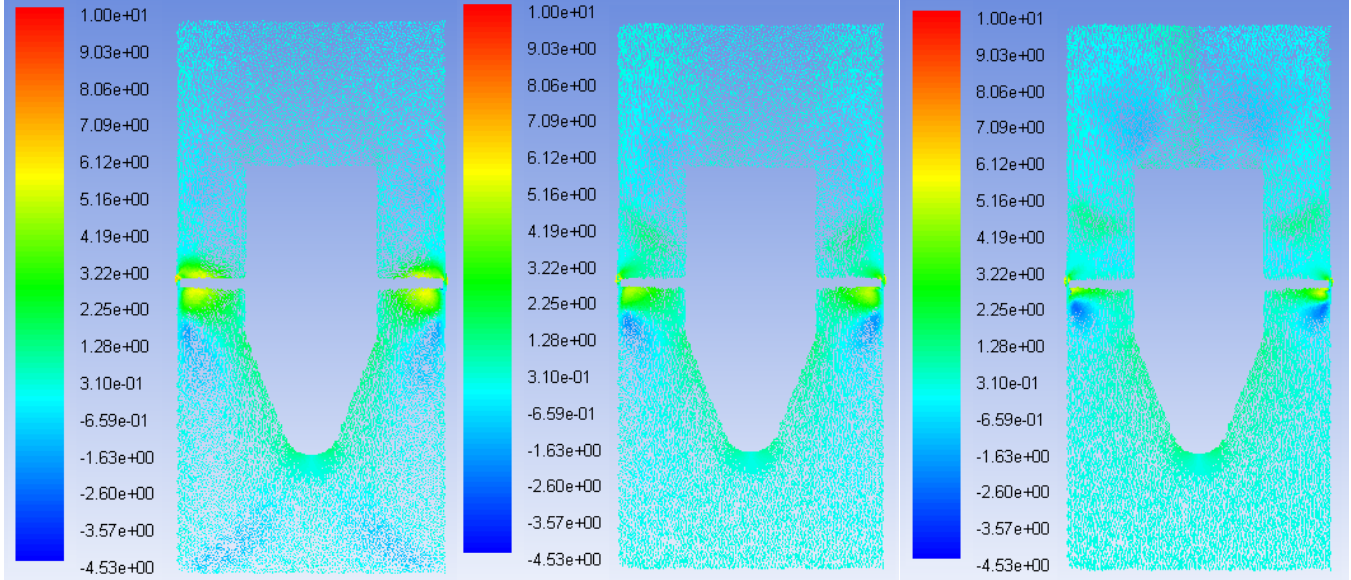

(B) Middle surface of radial velocity vectors

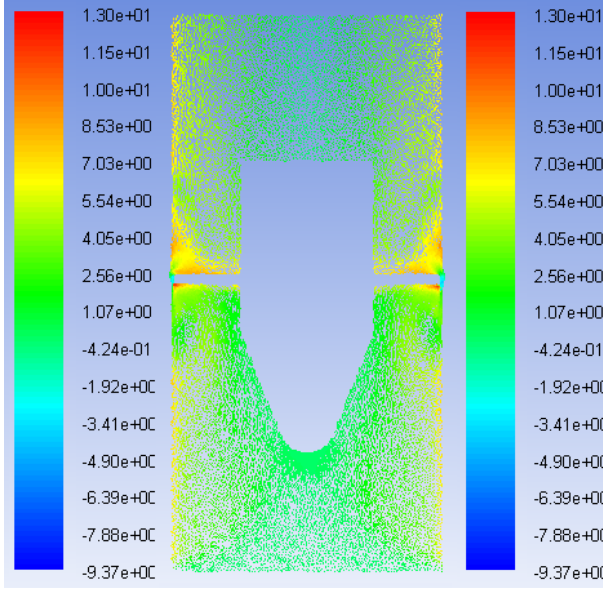

(a)

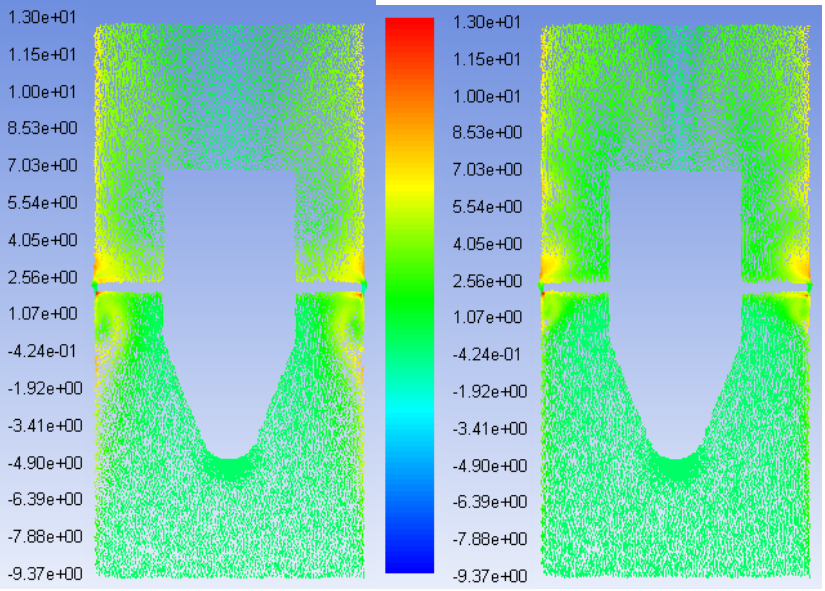

(b) (c)

Fig. 6. Middle surface of axial, radial and tangential velocities vectors under different flow rates

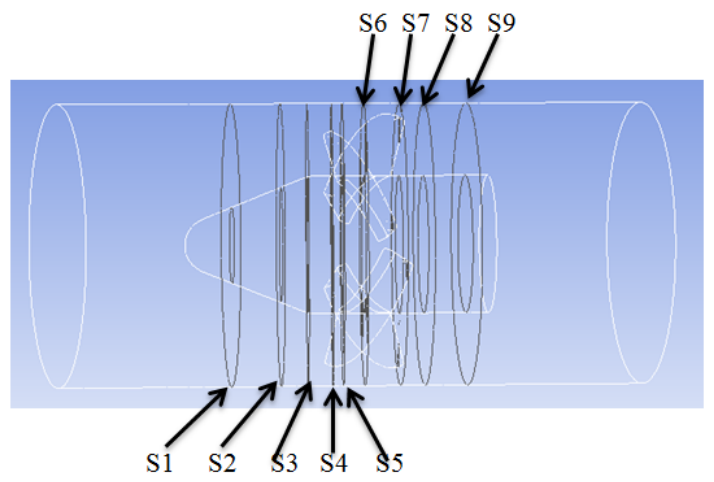

Fig. 7. Surface locations of static pressure on a pump
Figure 8 depicts variations of static pressure at nine surface locations on an impeller. Pump with 4 blades impeller diameter used is $102 \mathrm{~mm}$ and speed is $3000 \mathrm{rpm}$ with variety of flows. The surface locations (A) are named S1 to S3, and B) from $S 4$ to $S 6$ as well location $C$ ) from $S 7$ to $S 9$. Numerical results have shown that at surface locations (A) from $S 1$ to $S 3$ there is small change in the static pressure variations under different flow rates due to position of these locations are at the front of the axial impeller and at this region has low pressure variations. Also, it can be seen that at surface locations (B) from S4 to S4 there is clear change in the static pressure variations due to position of these locations are at middle and rear of the axial impeller, this region has high 
pressure variations. in addition, it is found that change in pressure variations increases as the rate of flow decreases and pressure variations are increase when the position of surface locations are near or closed to the middle surface location of the impeller of the axial pump. Furthermore, the surface locations $(\mathrm{C})$ from $\mathrm{S} 7$ to $\mathrm{S} 9$ has the same trend for the change in pressure variations as compared to the surface locations (B) but the pressure variations were decrease when the position of surface locations are far away from the middle surface location.
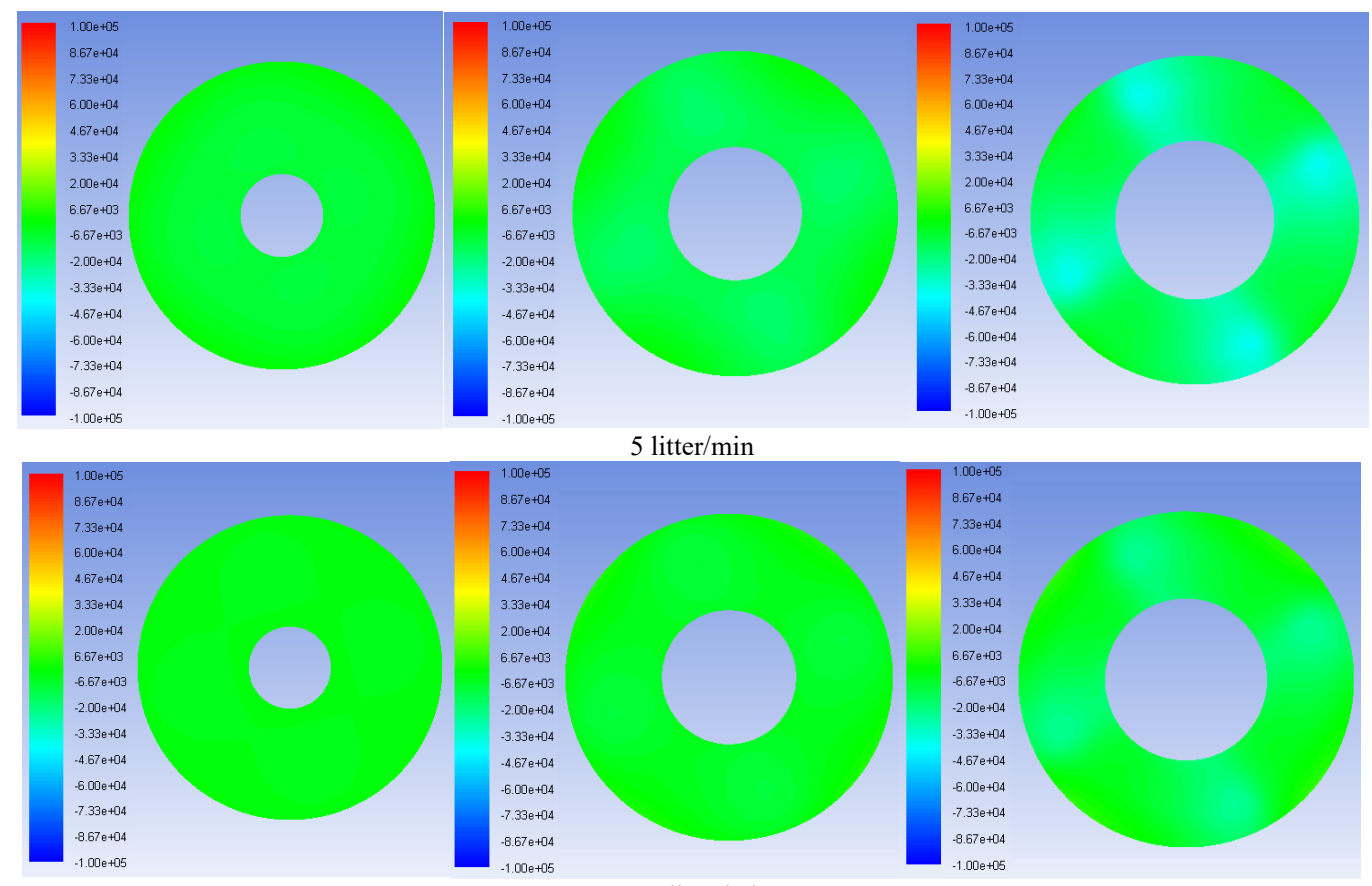

$12.5 \operatorname{litter} / \mathrm{min}$

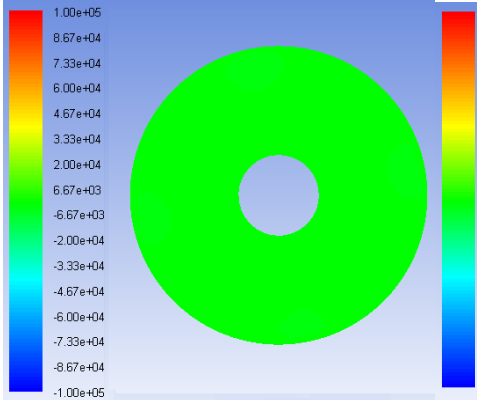

S1

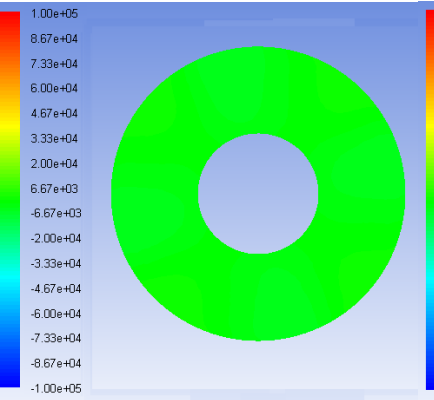

S2

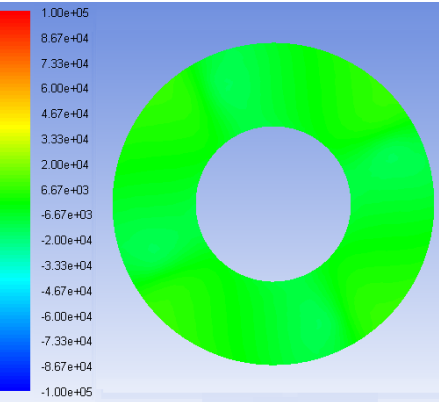

S3

20 litter/min

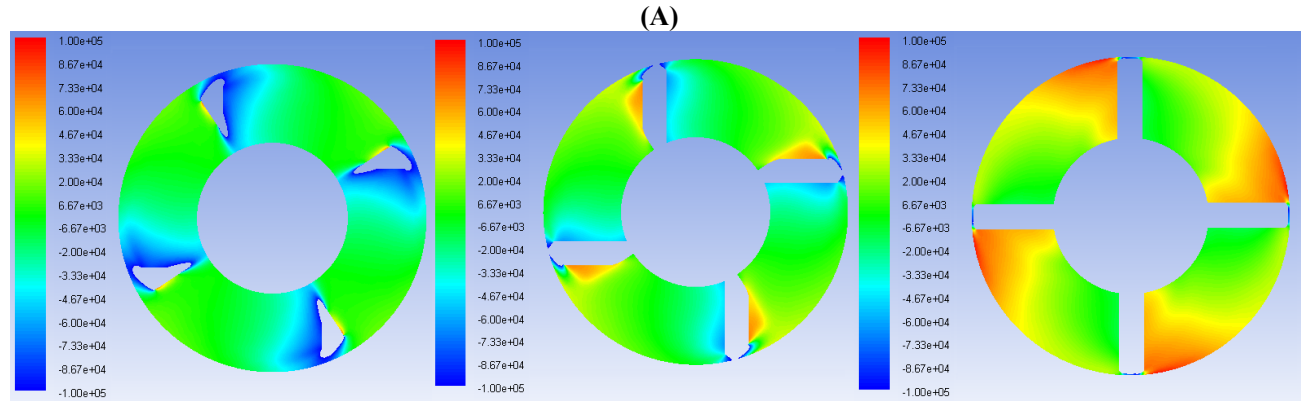

5 litter/min

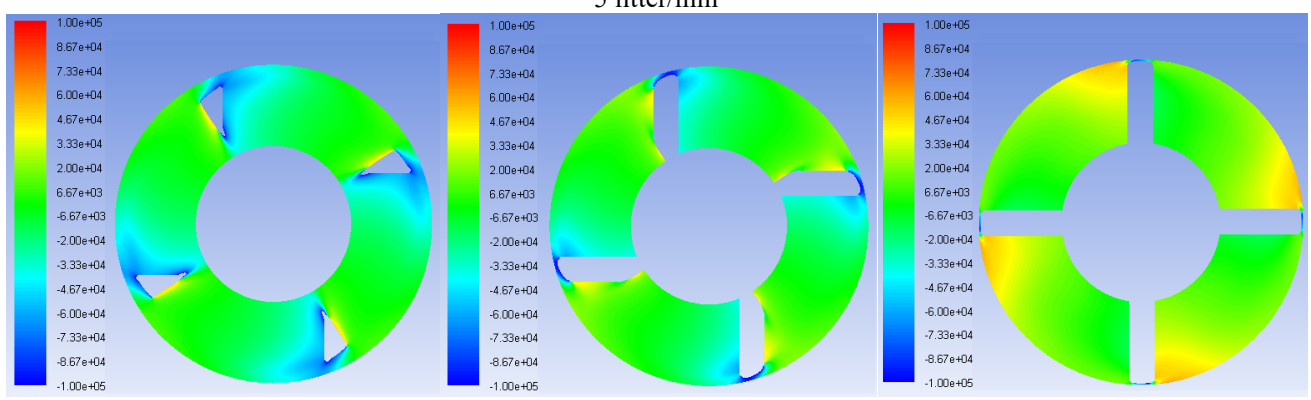

12.5 litter/min 

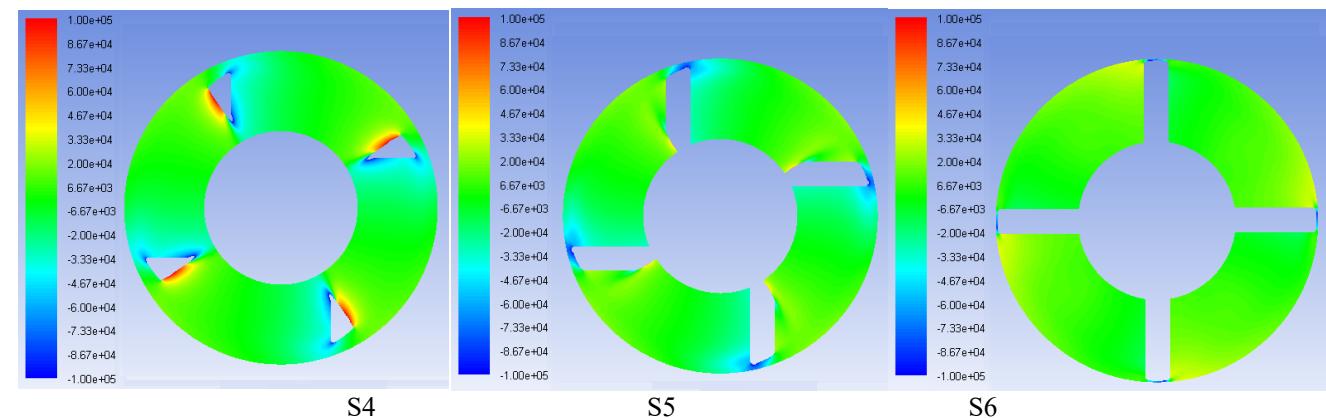

S5

S6

20 litter/min

(B)

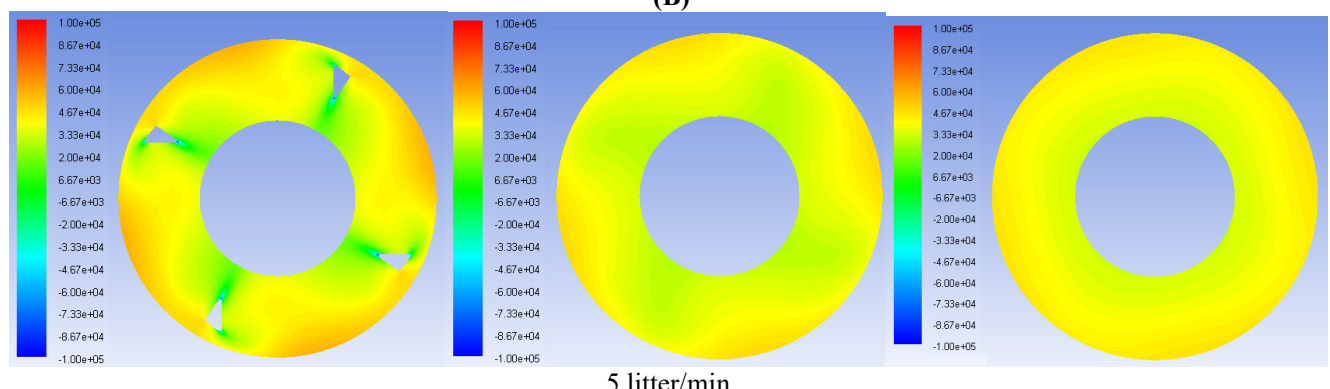

5 litter/min

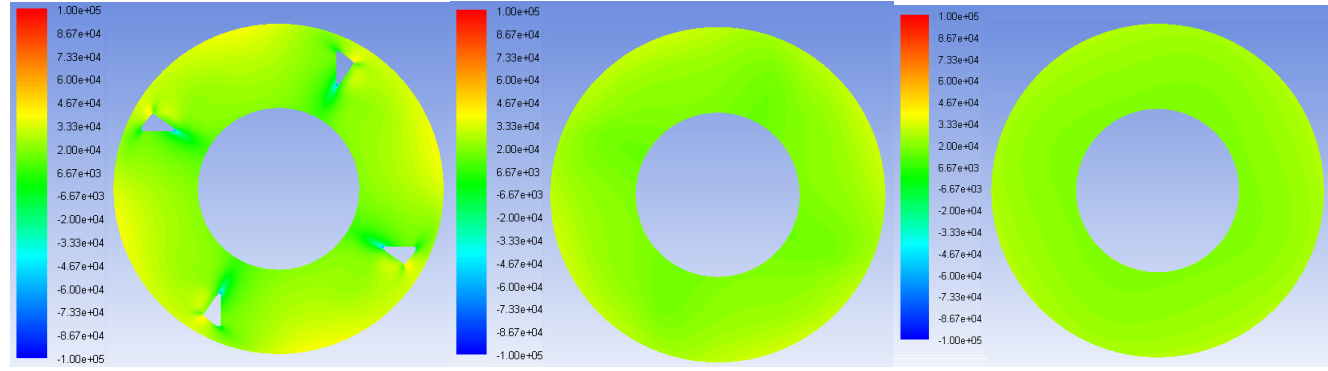

12.5 litter/min

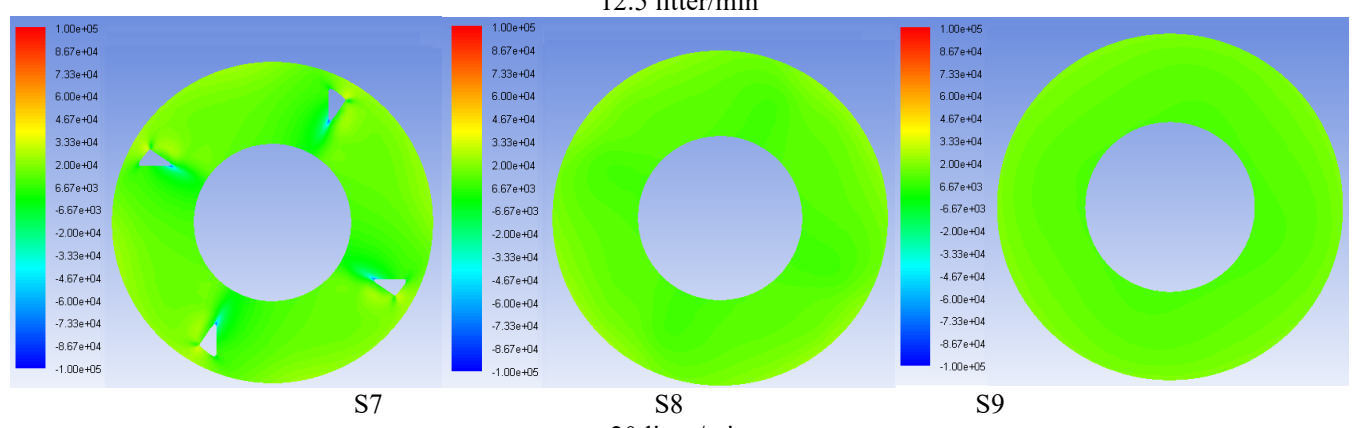

20 litter/min

(C)

Fig. 8. surface locations of static pressure on an axial flow impeller

\subsection{Monitoring Flow Points}

several points are positioned in the surface blade of axial impeller to analyse and understanding the fluctuations in pressure in a pump [9]. 36 points that are marked in the impeller blade. The monitoring points called 1 to 36 . As depicted in Figure 10 the location of these monitoring points. These monitoring points distributions to five ares on the blade the first one start from 1 to 6 , second one from 7 to 13 , third on from 14 to 19 , fourth one from 20 to 32 and last one from 33 to 36 as revealed in Figure 9. 


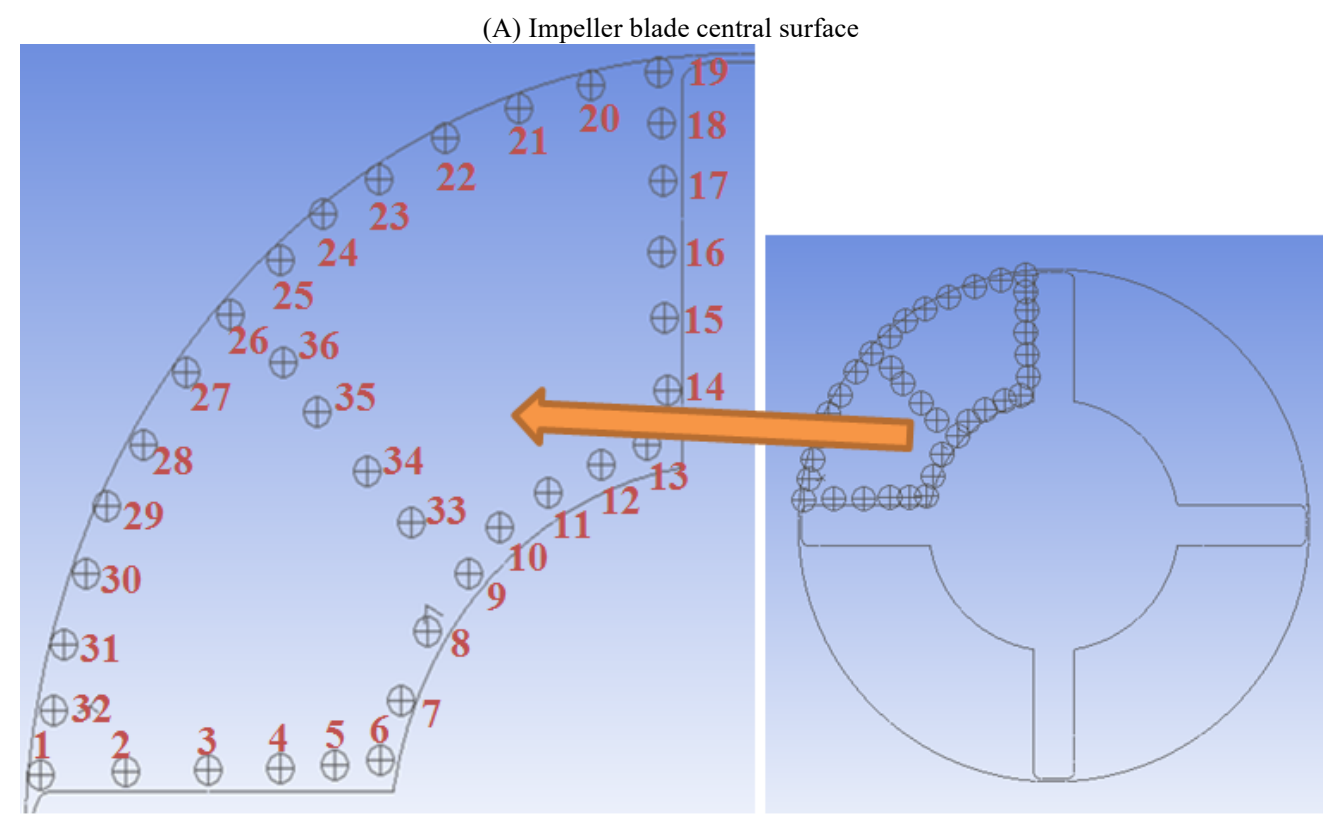

(B) monitoring locations points in a blade

Fig. 9. (A) Middle surface of the impeller blade, and (B) Location of points in a blade

\subsection{Blade Pressure Fluctuations Analysis}

Figure 10 represents pressure in the pump with 4 blades impeller and diameter is $102 \mathrm{~mm}$ with different rates of flow from 5 to 20 litter/min. This Figure depicts average value of pressure for point 1 with several flows. The trend of average pressure reduces as flow rate rises.

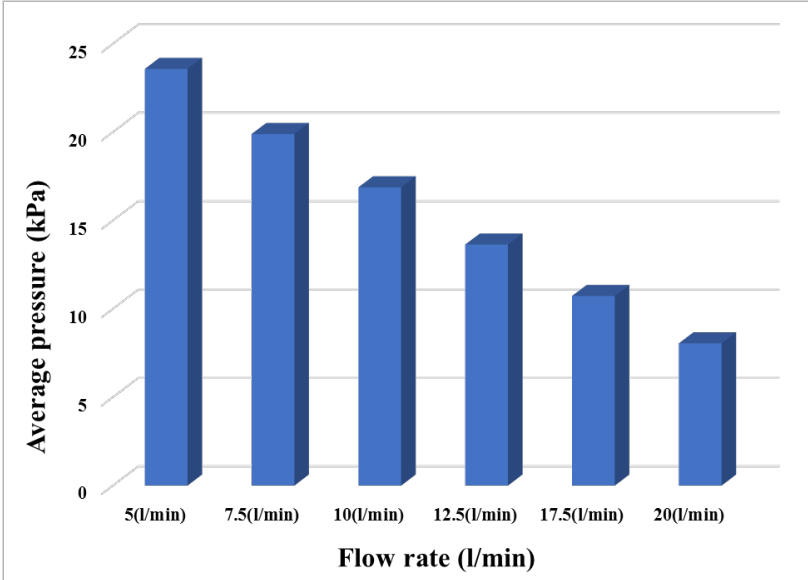

Fig. 10. Trend of pressure at various rate of flows

Figure 11 shows average pressure for area 1 on the blade of impeller and the monitoring point start from 1 to 6 . It can be shown that the average pressure for each monitoring point under different flows decreases when the flow rises. Also, there are positive pressure region and negative pressure region, due to this negative region of the blade located at lower pressure area. So, it is caused cavitation pfenomenon because of the decline in pressure in this special area.

To more analysis regarding pressure, Figure 12 describes the average pressure for area 2 on the blade of impeller and the monitoring point start from 7 to 13 . The average pressures in this eara have the similar tendency as compared to area 1. It is noticed that the average pressure rise between monitoring point 7 and 13 due to increase the pressure near the hub of an impeller. Also, there is negative pressure at location point 7 , owing to position this point was near lower pressure region.
Hence, may that leads to cause cavitation conditions in this particular region due to the same above reason.

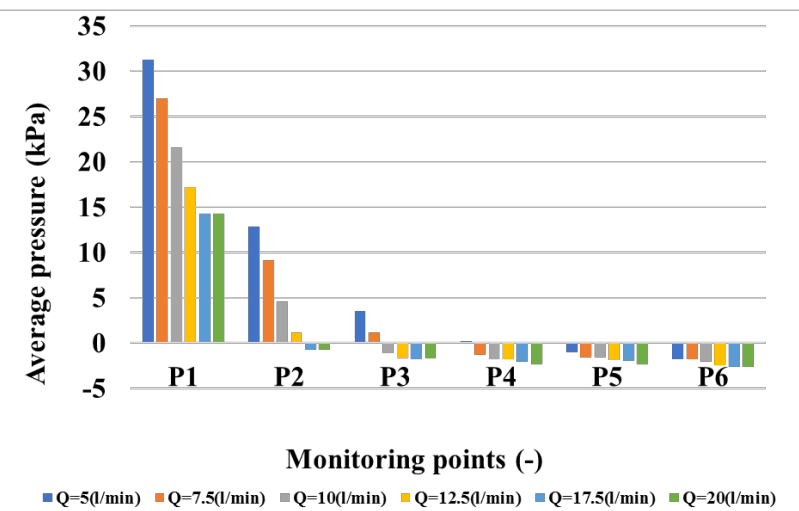

Fig. 11 Trend of average pressure for the monitoring points from 1 to 6

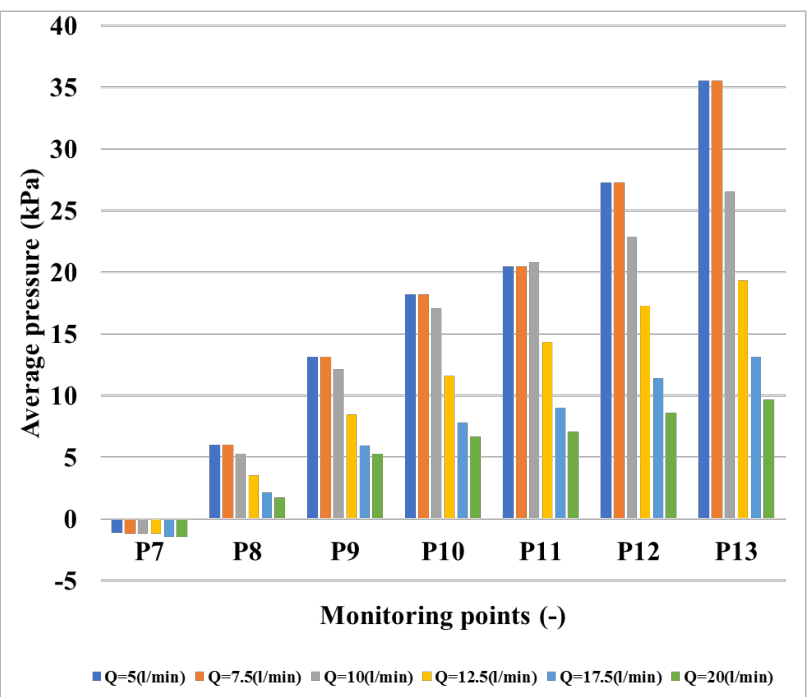

Fig. 12 Average pressure for the monitoring points from 7 to 13 
Figure 13 illustrates average pressure for area 3 on the blade of impeller and the monitoring point start from 14 to 19. It can be shown that the average pressure in this area also has the same trend as compared to area 2 . The average pressure increase from monitoring point 14 to 19 under different flow rates. there are different possible reasons firstly, it is owing to flow interaction for blades and flow interaction for stationary part and rotor part (impeller). Secondly, it is because of the location of these point are near high pressure on the impeller blade.

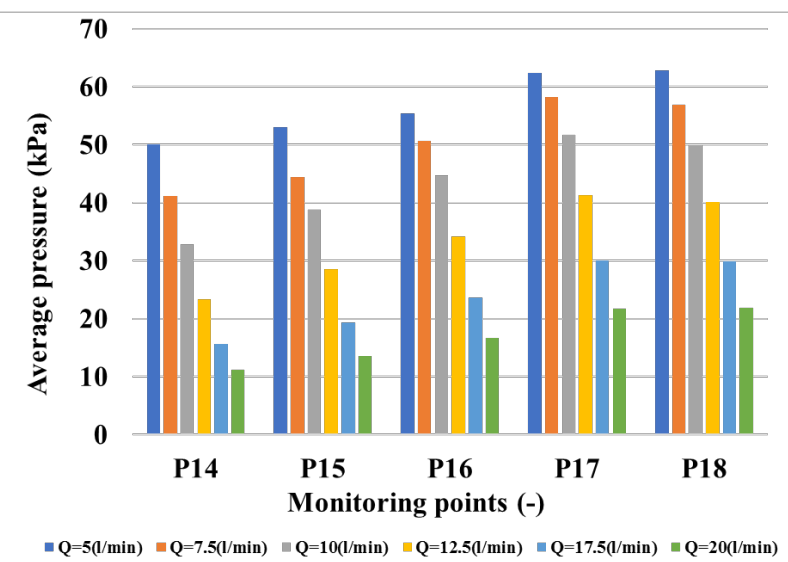

Fig. 13. Average pressure for the monitoring points from 14 to 19

Figure 14 shows average pressure for area 4 on the blade of impeller and the monitoring point start from 20 to 32. As noted that average pressure in this area also gradually decrease from monitoring point 20 to 32 under different flow rates. Futhermore, the numerical reslt have shown that the average pressure for monitoring point 20 has high pressure as compared to all monitoring point in this particular region due to location of this monitoring point was at high pressure at tip balde.

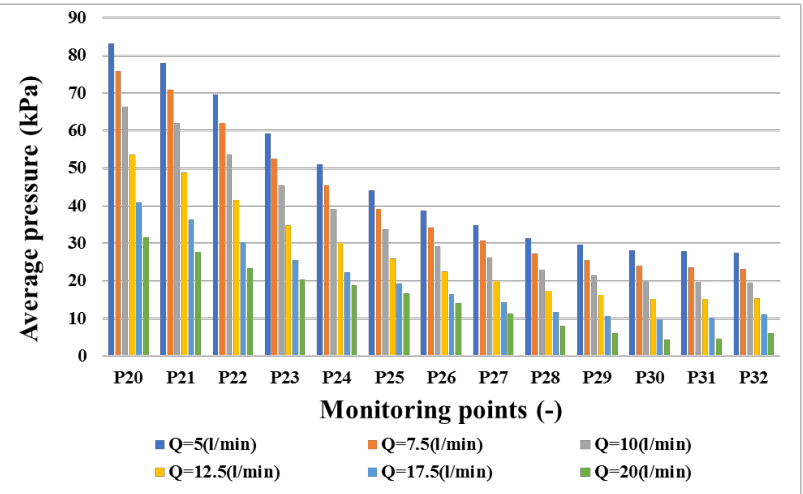

Fig. 14. Average pressure for the points between 20 and 32

Figure 15 displays average pressure with area 5 on the blade of impeller and the monitoring point start from 33 to 36 on the middel of blade surface. As observed that average pressure in this area increase gradually between monitoring point 33 and 36 at changed rate of flow. Futhermore, the monitoring point 36 under different flow rates has high pressure due to the poistion of this point from high intractions between the impeller and pipe wall.

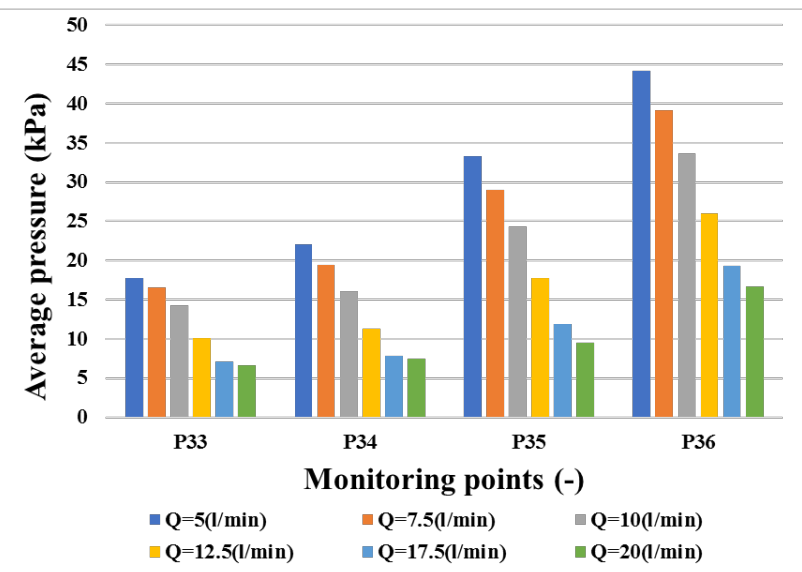

Fig. 15. Average pressure for the monitoring points from 33 to 36

\section{Conclusions}

According to the above computational calculations analysis on flow pattern and fluctuation in pressure analyses following conclusions can be found:The pressure in pump rises from region of impeller inlet to the impeller outlet region. The higher value of pressure was take placed near the impeller outer region.Pressure at the impeller blades suction surface was lower than pressure outlet impeller surface owing to the impact of pressure distribution. The numerical results noted that as the pump works at higher value of flows the area of high-pressure in impeller decreases. At low flow $(51 / \mathrm{min})$ in middle surface the high-speed area appears near or closed blade and blade tip region for all velocities under investigations.At surface locations (A) from $\mathrm{S} 1$ to $\mathrm{S} 3$ there is small change in the static pressure variations under different flow rates due to position of these locations are at the front of the axial impeller and at this region has low pressure variations. The surface locations (B) from S4 to S4 shows that there is clear change in the static pressure variations, due to position of these locations are at middle and rear of the axial impeller.The surface locations (C) from S7 to S9 has the same trend for the change in pressure variations as compared to the surface locations (B) but the pressure variations were decrease when the position of surface locations are far away from the middle surface location.Fluctuations in pressure for monitoring point at variety rate of flow was including 4 valleys and peaks with similar impeller blades number.The numerical results showed that different negative and positive pressure areas in the pump due to the varations in pressure.

\section{Acknowledgments}

The author necessity to thank Mustansiriyah University (www.uomustansiriyah.edu.iq) Baghdad - Iraq for its support in this work. Moreover, he also desires to thank University of Huddersfield.

This is an Open Access article distributed under the terms of the Creative Commons Attribution License

\section{References}

[1] Cong G H and Wang F J 2008 Transactions of the Chinese Society for Agricultural Machinery 39(6) 60-67.
[2] Al-Obaidi, A. (2018). Experimental and Numerical Investigations on the Cavitation Phenomenon in a Centrifugal Pump (Doctoral dissertation, University of Huddersfield). 
[3] Shi, L., Tang, F., Xie, R., \& Zhang, W. (2017). Numerical and experimental investigation of tank-type axial-flow pump device. Advances in Mechanical Engineering, 9(3), 1687814017695681

[4] Al-Obaidi, A. R. (2019). Effects of different turbulence models on three-dimensional unsteady cavitating flows in the centrifugal pump and performance prediction. International Journal of Nonlinear Sciences and Numerical Simulation, 20(3-4), 487-509.

[5] Al-Obaidi, A. R. (2019, July). Numerical Investigation of Flow Field Behaviour and Pressure Fluctuations within an Axial Flow Pump under Transient Flow Pattern Based on CFD Analysis Method. In Journal of Physics: Conference Series (Vol. 1279, No. 1, p. 012069). IOP Publishing.

[6] Shi, L., Tang, F., Xie, R., \& Zhang, W. (2017). Numerical and experimental investigation of tank-type axial-flow pump device. Advances in Mechanical Engineering, 9(3), 1687814017695681..

[7] Meng, F., Yuan, S., \& Li, Y. (2018). Fluid-structure coupling analysis of impeller in unstable region for a reversible axial-flow pump device. Advances in Mechanical Engineering, 10(3), 1687814017751762

[8] Pan, H., Zhang, F., \& Zheng, Y. (2018, July). Pressure fluctuation analysis of axial-flow pump based on local mean decomposition. In IOP Conference Series: Earth and Environmental Science (Vol. 163, No. 1, p. 012124). IOP Publishing.

[9] Song, X. J., \& Liu, C. (2018, July). Numerical analysis of the influence of vortex on the pressure pulsation in axial flow pump system based on the CFD. In IOP Conference Series: Earth and Environmental Science (Vol. 163, No. 1, p. 012106). IOP Publishing.

[10] Bosioc, A. I., Muntean, S., Draghici, I., \& Anton, L. E. (2016, November). Hydrodynamic Analysis of the Flow in an Axial Rotor and Impeller for Large Storage Pump. In IOP Conference Series: Earth and Environmental Science (Vol. 49, No. 3, p. 032016). IOP Publishing.

[11] Cheng, L., Liu, C., Luo, C., Zhou, J. R., \& Jin, Y. (2012). Research on the unstable operating region of axial-flow and mixed flow pump. In IOP Conference Series: Earth and Environmental Science (Vol. 15, No. 3, p. 032050). IOP Publishing.

[12] Pan, Z., Ni, Y., Yuan, J., \& Ji, P. (2015). Hydraulic Performance Comparison for Axial Flow Impeller and Mixed Flow Impeller with
Same Specific Speed. In Journal of Physics: Conference Series (Vol 656, No. 1, p. 012068). IOP Publishing.

[13] Tao, R., Xiao, R., Yang, W., Wang, F., \& Wu, Y. (2013). Investigation of the Hydrodynamics of Sweep Blade in Hi-Speed Axial Fuel Pump Impeller. Advances in Mechanical Engineering, 5, 174017.

[14] Li, W. Y., Zhang, X. Y., Shuai, Z. J., Jiang, C. X., \& Li, F. C. (2014). CFD numerical simulation of the complex turbulent flow field in an axial-flow water pump. Advances in Mechanical Engineering, 6, 521706.

[15] Mostafa, N. H., \& Mohamed, A. (2012). Effect of blade angle on cavitation phenomenon in axial pump. Journal of Applied Mechanical Engineering, 3(1), 1-6.

[16] Al-Obaidi, A. R. (2019). Monitoring the performance of centrifugal pump under single-phase and cavitation condition: A CFD analysis of the number of impeller blades. J. Appl. Fluid Mech, 12(2), 445459.

[17] Hua, T., L. Yi, and Z. Yu-Liang, Numerical analysis of a prototype centrifugal pump delivering solid-liquid two-phase flow. Journal of Applied Sciences, 2013. 13(17): p. 3416.

[18] Al-Obaidi, A. R. (2019). Investigation of effect of pump rotational speed on performance and detection of cavitation within a centrifugal pump using vibration analysis. Heliyon, 5(6), e01910.

[19] Al-Obaidi, A. R. (2019). Experimental investigation of the effect of suction valve opening on the performance and detection of cavitation in the centrifugal pump based on acoustic analysis technique. Archives of Acoustics, 44(1), 59-69.

[20] Liu, Y., Zhou, J., \& Zhou, D. (2017). Transient flow analysis in axial-flow pump system during stoppage. Advances in Mechanical Engineering, 9(9), 1687814017723280.

[21] Al-Obaidi, A. R. (2019). Investigation of fluid field analysis, characteristics of pressure drop and improvement of heat transfer in three-dimensional circular corrugated pipes. Journal of Energy Storage, 26, 101012.

[22] Al-Obaidi, A. R., \& Towsyfyan, H. (2019). An Experimental Study on Vibration Signatures for Detecting Incipient Cavitation in Centrifugal Pumps Based on Envelope Spectrum Analysis. Journal of Applied Fluid Mechanics, 12(6), 2057-2067. 